\title{
PROBLEMAS DE REEMPLAZO: ENFOQUES DE INVESTIGACIÓN DE OPERACIONES Y ECONOMÍA
}

\author{
Berger Vidal Esther, Olazo Carlos Julio, Oré Luján José C. ${ }^{1}$
}

\begin{abstract}
RESUMEN.- Se realiza el análisis de los enfoques de Investigación de Operaciones y Económico para la generación de alternativas que permitan tomar la política más adecuada de reemplazo de bienes, utilizados en los procesos de producción. Se presenta una clasificación de modelos de reemplazo usados por la Investigación de Operaciones los que buscan directamente optimizar variables de costo e indirectamente optimizar la productividad. Se presenta y analiza el enfoque y modelos usados por la Economía para el análisis de reemplazo.
\end{abstract}

\section{INTRODUCCION}

La definición de políticas de reemplazo de bienes, cuyo valor se deprecia en el tiempo al ritmo de la decadencia de su vida útil o tendencia a la obsolescencia, es muy importante para las organizaciones. Esta importancia radica en la necesidad de optimizar el rendimiento de los bienes, lograr retener el bien y asumir sus costos de mantenimiento en cuanto resulte beneficioso, teniendo en consideración que los costos de mantenimiento y operación de bienes se incrementan con la edad de estos.

La influencia de los costos de mantenimiento convierte un problema simple de reemplazo de un bien cuando falla o cuando acaba su vida útil, en un problema más complejo de reemplazo ya que si no existiera la necesidad de hacer mantenimiento bastaría con substituir el bien en el momento que falla o en el momento que se hace obsoleto.

Es importante indicar que reemplazar un bien puede significar sustituirlo por otro igual o no, es posible sustituirlo por otro similar, otro cuya tecnología es más moderna o por otro totalmente diferente al original, Bealey [3], Villaplana [7]. También es importante señalar que el análisis de reemplazo que con frecuencia se realiza a equipos, puede aplicarse a procesos, tecnologías, software o plantas físicas. En particular, en este caso nos referiremos al reemplazo de equipos.

Debe considerarse que, si una empresa no controla la decadencia de la capacidad productiva de sus equipos para determinar con oportunidad el fin de su servicio y su desplazamiento por otros, está recargando costos a su producción, los que se reflejarán en el costo final del producto y en la menor calidad de este. Como consecuencia, la productividad y competitividad de la empresa disminuyen. Si este escenario se produce en un país; la economía y la competitividad de éste se ven seriamente afectadas, Villaplana [7]. Lamentablemente este escenario es común en los países emergentes.

${ }^{1}$ Docentes del Departamento Académico de Investigación Operativa, de la Facultad de Ciencias

Matemáticas - UNMSM 
En este artículo presentaremos los enfoques que aplican la Investigación de Operaciones y la Economía para resolver el problema de decidir respecto al reemplazo de un equipo o establecer una política adecuada de reemplazo en un horizonte determinado de tiempo. El análisis comparativo de las tendencias en los resultados brindados por los enfoques de Investigación de Operaciones y de Economía forma parte de un estudio en proceso que someteremos a consideración en una próxima oportunidad.

\section{EL PROBLEMA DE REEMPLAZO}

Dado un equipo cuya edad en la fecha de adquisición es cero, se trata de determinar la edad del equipo que se considera conveniente substituir tomando en consideración las variables de tiempo de falla, costo inicial, gastos de operación, costo de mantenimiento, rendimiento del equipo y el valor de salvamento. El objetivo es minimizar el costo total o maximizar la productividad del equipo durante un horizonte de tiempo. Cuando se considera el valor del dinero en el tiempo se incorpora en el problema la tasa de actualización correspondiente. Existen dos formas de tratar el problema de reemplazo, uno desde el punto de vista de la Investigación Operativa y otro desde la Ėconomía. A continuación presentamos ambos enfoques.

\subsection{Enfoques de Investigación de Operaciones}

La Investigación de Operaciones utiliza diferentes herramientas para dar solución a estos problemas. Es posible enfocar los problemas de reemplazo alternativamente mediante la teoría de grafos y programación dinámica como enfoques determinísticos mediante el análisis de procesos estocásticos, programación dinámica y simulación de sistemas como enfoques estocásticos.

\subsubsection{Enfoque de Grafos}

Se plantea un problema de camino mínimo o ruta más corta. Se consideran el horizonte de tiempo, el costo de compra del equipo en el año j, el valor de salvamento del equipo después de j años de uso, así como el costo de mantenimiento y operación.

Sea un equipo cuyo análisis de reemplazo se realizará bajo las siguientes condiciones:

Horizonte de tiempo: $\mathrm{T}$ años.

$P_{j}$ : Valor, precio de compra del equipo o costo del equipo al inicio del año $j$

$C_{j}$ : Costo de mantenimiento y operación durante el año $j$, tal que $C_{j+1}>C_{j}$ para $\mathrm{j}=1,2,3, \ldots, \mathrm{T}$.

$S_{j}: \quad$ Valor de salvamento en el año $j$ 
Se trata de determinar la política óptima de reemplazo del equipo con el objetivo de minimizar el costo total, considerando que no hay variación en el valor de estos factores a través del tiempo.

Para formularlo como un problema de ruta más corta se construye un grafo orientado y acíclico $\mathrm{G}=(\mathrm{N}, \mathrm{A})$, tal que el conjunto de nodos $\mathrm{N}$ se define del siguiente modo:

$\mathrm{N}=\{0,1,2,3, \ldots, \mathrm{T}\}$, donde el nodo 0 es el inicio del horizonte que representa la fecha de adquisición y el nodo j es el inicio del período j ó fin del período $\mathrm{j}-1$, que representan fechas en las cuales es posible reemplazar el equipo

$$
A=\{\operatorname{arcos}(i, j) / j>i\} .
$$

Donde $\mathrm{j}>\mathrm{i}$ expresa que si se reemplazó el equipo en el período i un nuevo reemplazo sólo podrá ocurrir posteriormente, en un período j.

La medida de distancia entre dos nodos i y j estará dada por la variable $\mathrm{D}_{\mathrm{ij}}$ que representa el costo de compra, más el costo de mantenimiento y operación, menos el valor de salvamento calculados al inicio del año i para reemplazarlo al inicio del año j.

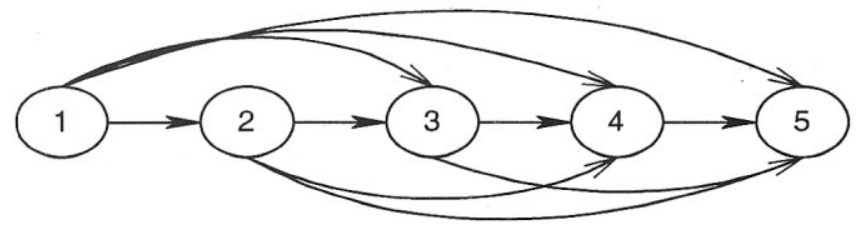

De este modo, tenemos:

$$
\begin{gathered}
D_{i j}=P_{j}+\sum_{i-j}^{j-i} C_{i}-S_{j-i} \text { cuando } j>i \\
D_{i j}=\infty \text { cuando } j \leq i(\text { no existe arco de la } i \text { a } j \text { ) }
\end{gathered}
$$

Para resolver este problema es aplicable el Algoritmo de Dijkstra. La secuencia de arcos en el camino o ruta que hallará el algoritmo constituye la política óptima de reemplazo del equipo definida por la ruta de menor distancia entre el nodo inicial y el final del grafo, representada por el costo total.

\subsubsection{Enfoque de Programación Dinámica Determinística}

También es posible formular un modelo de programación dinámica en el cual desde un estado posible que significa «equipo nuevo» en la etapa i es posible pasar a un nuevo estado "equipo nuevo" en la etapa $(\mathrm{i}+\mathrm{k})$ y con la información de costos de mantenimiento, operación y reemplazo, y el valor de salvamento determinar la política óptima asociada al mínimo costo. "equipo nuevo" al inicio de la etapa i es equivalente a "reemplazo del equipo antiguo" al final de la etapa i - 1 . 
Sea un equipo cuyo análisis de reemplazo se realizará bajo las siguientes condiciones:

Horizonte de tiempo: T períodos.

$\mathrm{P}_{\mathrm{j}:} \quad$ Valor, precio de compra o costo del equipo al inicio del período j.

$C_{j}$ : Costo de mantenimiento y operación durante el año $j$, tal que $C_{j+1}>C_{j}$ para $\mathrm{j}=1,2,3, \ldots, \mathrm{T}$.

$S_{j}: \quad$ Valor de salvamento.

$\mathrm{d}_{1}$ : Decisión de reemplazar el equipo al final de un período.

$\mathrm{d}_{2}$ : Decisión de no reemplazar el equipo al final de un período.

Se trata de determinar la política óptima de reemplazo del equipo con el objetivo de minimizar el costo total, considerando que no hay variación en el valor de estos factores en el tiempo, para esto se define:

f (t) : Costo neto mínimo generado desde $t$ hasta $T$; dado que se compró un equipo nuevo al inicio del período $t$, es decir, dada la decisión $\mathrm{d}_{1}$. El costo neto incluye el costo de compra y el valor de salvamento del equipo recientemente adquirido.

$C_{\mathrm{tx}}$ : Costo neto que incluye el costo de compra y el valor de salvamento, por comprar un equipo al inicio del período $t$, cuya vida útil es $t+x$. Esto significa que el equipo se utilizará hasta el período $\mathrm{x}$.

Tenemos la fórmula recursiva de programación dinámica, para el procedimiento de solución de adelante hacia atrás:

$$
f(t)=\underset{x=t+1, t+2, \ldots, T}{\text { Mínimo }}\left\{C_{t x}+f(x)\right\} ; t=T-1, T-2, \ldots ., 1
$$

De este modo, si la máquina se reemplaza en un período $x$ cualesquiera, debemos determinar el costo desde el período t hasta $\mathrm{T}$, el cual está dado por el costo total desde que se compra el equipo hasta que se reemplaza o vende en el período $x$, es decir, $C_{t x}$, más el costo total desde el período $x$ hasta $T$, representado por $f(x)$.

Si se toma la decisión $\mathrm{d}_{2}$, de no reemplazar el equipo, este continuará operando ocasionando costos de mantenimiento y operación que serán tomados en consideración cuando se tome la decisión $\mathrm{d}_{1}$. Así, el problema consiste en escoger adecuadamente $x$ tal que minimice la suma de costos, tal como se expresa en (2).

La representación gráfica del problema lleva al mismo grafo presentado en (3.1). El procedimiento recursivo de solución que realiza la programación dinámica es equivalente a hallar la ruta más corta desde el nodo inicial hasta el nodo $\mathrm{T}$, representando $\mathrm{C}_{\mathrm{ij}}$ la longitud del arco entre i y j. 
El planteamiento presentado considera el valor del dinero estable o constante. Para considerar el valor real del dinero en el tiempo, que permita ponderar adecuadamente los costos o beneficios futuros, se asigna pesos o ponderaciones a cada uno de estos factores, dando mayor ponderación al costo o beneficio más reciente y disminuyéndola gradualmente, de acuerdo al problema, conforme el período futuro se aleja del presente.

Sea $0<\delta<1$ una ponderación, la cual representa una proporción del valor de $f(x)$ al final del período $x$, medido al inicio del período $x$. Incorporando este factor a la fórmula recursiva de programación dinámica tenemos:

$$
\mathrm{f}(\mathrm{t})=\underset{\mathrm{x}=\mathrm{t}+1, \mathrm{t}+2, \ldots, \mathrm{T}}{\operatorname{Minimo}}\left\{\mathrm{C}_{\mathrm{tx}}+\delta \mathrm{f}(\mathrm{x})\right\} ; \mathrm{t}=\mathrm{T}-1, \mathrm{~T}-2, \ldots, 1 ; 0<\delta<1
$$

\subsubsection{Análisis de Procesos Estocásticos}

En el enfoque probabilístico de Investigación de Operaciones, nuestra propuesta es que si se dan las condiciones, esto es, si se dispone de la información suficiente y adecuada para representar el problema como un proceso estocástico, podemos considerar el espacio paramétrico $T$, discreto, tal que $T=\{t: 0,1,2$, $3, \ldots ., \mathrm{M}\}$ y el espacio de estados discreto $S=\{x$ : vida ủtil restante tal que $x=0$, $1,2,3, \ldots, \mathrm{s}$ períodos\}. En este caso, es posible tratar el problema como una cadena de Markov para lo cual deben cumplirse las dos propiedades siguientes:

1 ) Propiedad de Markov: Para todo n se cumple:

$$
P\left(X_{n}=j_{n} / X_{n-1}=j_{n-1}, X_{n-2}=j_{n-2}, \ldots, X_{1}=j_{1}, X_{0}=j_{0}\right)=P\left(X_{n}=j_{n} / X_{n-1}=j_{n-1}\right)
$$

2 ) El proceso es estacionario, es decir que para todo m y n se cumple:

$$
P\left(X_{n}=j / X_{n-1}=i\right)=P\left(X_{m}=j / X_{m-1}=i\right)
$$

Las probabilidades de transición de un paso están dadas por:

$\mathrm{p}_{\mathrm{ij}}=\left\{\mathrm{X}_{1}=\mathrm{j} / \mathrm{X}_{0}=\mathrm{i}\right\}$, donde $\mathrm{p}_{\mathrm{ij}}$ expresa la probabilidad de pasar del estado inicial vida útil de longitud $i$ al estado vida útil restante de longitud $j$ en el período inmediatamante siguiente, con $\mathrm{i}>\mathrm{j}$.

La matriz de transición $\mathbf{P}$ cuyos elementos son las $\mathrm{p}_{\mathrm{ij}}$ expresa las probabilidades de todos los cambios de estado vida útil restante de un período a otro inmediatamente siguiente. $\mathbf{P}$ es la matriz de probabilidades de transición de un paso que muestra el proceso de disminución de la vida útil entre dos períodos consecutivos.

Si el equipo es sometido a una operación de mantenimiento al inicio de un período determinado puede considerarse una prolongación de la vida útil restante, asignando una probabilidad mayor que cero a la posibilidad de mantener un período adicional de vida útil restante. Es decir, si en un período $\mathrm{r}$ determinado el equipo tiene como vida útil restante $\mathrm{k}$ períodos y se le da mantenimiento, es posible que nuevamente le queden $\mathrm{k}$ períodos de vida útil (no envejeció), significando un cambio virtual de estado, representable mediante un lazo en un grafo de transición de estados, lazo al cual se asocia una probabilidad mayor que cero que, sin embargo, es muy pequeña. 
Esto es aceptable siempre que el costo de mantenimiento y operación hasta el final del horizonte de tiempo no superen el rendimiento económico del equipo. En caso contrario, no debe considerarse la posibilidad de volver al mismo estado.

Tomando en consideración que el análisis del proceso parte del instante de la compra del primer equipo, se iniciará el desarrollo del proceso con el vector de probabilidades de estado:

$$
\mathrm{p}=\left(\mathrm{p}_{1}, \mathrm{p}_{2}, \ldots . ., \mathrm{p}_{\mathrm{s}}\right)=(1,0, \ldots, 0)
$$

La matriz de transición de $n$ pasos $\mathbf{P}^{(\mathrm{n})}$ expresa la probabilidad de pasar del estado vida útil restante de i períodos al estado vida útil restante de j períodos. Estamos considerando que el cambio de la vida útil restante del equipo no necesariamente disminuye uno a uno, pudiendo darse en saltos mayores cuando la gravedad de las fallas en las componentes o en el equipo total así lo determinan.

También es posible pasar de una vida útil restante mayor que cero a una vida útil restante igual a cero. Esto se presenta cuando en el sistema ocurre una falla total no recuperable o cuyo mantenimiento producirá mayores costos que beneficios generados debido al rendimiento futuro del equipo.

Las Probabilidades de Estado Estable definidas por el vector $\Pi$ indican la probabilidad a largo plazo de estar en un estado vida útil restante determinado. En el largo plazo, no se consideran cambios virtuales de estado, siendo $\Pi=(0,0, \ldots ., 1)$ debido a que la vida útil restante va disminuyendo su valor hasta cero conforme se incrementa el tiempo. Este resultado está asegurado debido a que el estado vida útil restante mínima o cero es el único estado absorbente y a que la estructura del proceso garantiza que se ingresará a éste en un número finito de pasos. El diagrama de transición de estados, es:

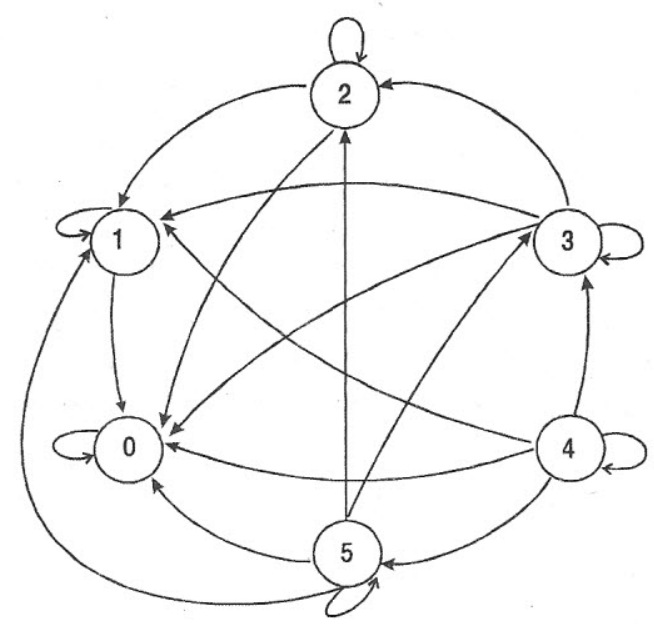

Los resultados asociados a datos de costos permiten determinar cuánto tiempo es conveniente mantener un equipo o cuándo substituirlo. En este caso se involucran costos de operación, costos de mantenimiento y costos de penalización cuando el equipo falla y es reparado; luego, se disminuye de estos costos el valor de salvamento. De este modo debemos determinar la política óptima de reemplazo, teniendo en consideración el costo total ( CT ) ante diferentes alternativas aleatorias que el diagrama de cambio de estados o la matriz $\mathbf{P}$ permiten visualizar; 


$$
C T=\left(\frac{1}{n}\right) \sum_{i=1}^{n}\left(c r_{i}+c o_{i}\right) p_{i}-S
$$

Donde:

$\mathrm{cr}_{\mathrm{i}}$ : Costo de penalización por falla por realizar mantenimiento o reparación cuando el equipo tiene una vida útil restante de i períodos; $\mathrm{cr}_{\mathrm{i}} \geq 0$.

$\mathrm{Co}_{\mathrm{i}}$ : Costo de operación durante el período i

$S$ : Valor de salvamento

$\mathrm{p}_{\mathrm{i}}$ : Probabilidad del estado $\mathrm{i}$.

No se plantean los costos esperados para las probabilidades de estado estable, dado que sólo se evaluaría para el estado absorbente y no se estarían reflejando los costos reales del proceso.

El análisis de costos esperados debe desarrollarse evaluando diferentes caminos alternativos para llegar al estado absorbente y elegir aquella alternativa que arroje el menor costo promedio total.

\subsubsection{Simulación de problemas de reemplazo}

Simular un problema de reemplazo implica conocer la distribución de probabilidad de falla asociada a la variable aleatoria $T$; la cual representa el tiempo desde un instante $t=0$ en que se inicia un período de funcionamiento del equipo, hasta que ocurre una falla. $\mathrm{f}(\mathrm{t})$ representa la función densidad de probabilidad de $T$. F ( $t$ ) representa la probabilidad de una o más fallas hasta el período $t$. $1-\mathrm{F}(\mathrm{t})$, conocida con el nombre de función de confiabilidad, es la probabilidad de que el equipo funcione un período mayor que $t$.

Mediante simulación podemos analizar el proceso aleatorio de ocurrencia de fallas que darán lugar a acciones de reparación. Una operación de mantenimiento puede ser rutinaria, es decir, no requiere la ocurrencia de falla y ocasiona un costo $\mathrm{cm}_{\mathrm{i}}$ cuando se realiza en el período i. Una acción de reparación que obviamente sólo se realiza cuando se presenta falla, puede durar uno o más períodos e implica un costo de reparación por período $\mathrm{cr}_{\mathrm{i}}$ y un costo de penalización $\mathrm{cp}_{\mathrm{i}}$ por la falta de funcionamiento del equipo, durante cada período que este parado. A estos costos se agrega el costo inicial CI de compra e instalación y se resta el valor de salvamento $\mathrm{S}$ al final del horizonte de tiempo.

Los componentes del Modelo de Simulación son:

Variables Exógenas : Tiempo de falla.

Variables Endógenas: Número total de reparaciones, número total de fallas, número total de mantenimientos, costos totales, costos promedio finales.

Variables de Estado : Tiempo funcionando, tiempo sin funcionar, tiempo en mantenimiento, tiempo en reparación.

Parámetros : Estados posibles, costo de mantenimiento por período, costo de reparación. 
En este proceso se puede comenzar desde la alternativa más temprana de reemplazo, evaluando el costo y el rendimiento económico del equipo, continuándose consecutivamente con la segunda alternativa más temprana, evaluando el respectivo costo y rendimiento, de todas las alternativas. La simulación permite evaluar con facilidad todas las alternativas posibles y mostrar la alternativa de costo total mínimo dada por la secuencia de decisiones de reemplazo. Este es una análisis de optimización mediante simulación.

Las variables endógenas permiten analizar tanto los resultados de la operación del sistema como los diferentes costos en que se incurre durante un horizonte de tiempo determinado. De este modo, apoya a las decisiones de reemplazo, no sólo a través de la información sobre los costos incurridos sino acerca del rendimiento del equipo en volumen de producción, tiempos de producción o tiempos muertos de producción debido a los períodos de reparación.

En este contexto, nos preguntamos si las alternativas que brinda la Investigación de Operaciones ofrecen al decisor un escenario de solución suficientemente amplio respecto al tipo y cantidad de variables que este requiere involucrar en su decisión. Esto nos lleva a analizar los enfoques comunmente usados en la Economía.

\subsection{Enfoques Utilizados en la Economía}

Para el enfoque económico y financiero es posible realizar el análisis de reemplazo antes o después de la vida útil esperada. El resultado básico de un análisis de reemplazo consiste en determinar si ya se alcanzó el final de la vida útil o simplemente vida útil de un activo y cuál será la alternativa que puede aceptarse para su reemplazo. La teoría y casos presentados se basan en información de Blank y Tarkin [2].

Desde el punto de vista de la economía el reemplazo se considera por diferentes razones:

i ) Cuando el desempeño ha disminuido debido al deterioro de las componentes y cuando la productividad medida en términos de la calidad y cantidad han disminuido significativamente. Debido a esto se incrementan los costos de operación, el desperdicio, los costos de reelaboración, las pérdidas en las ventas y los costos de mantenimiento.

ii) Cuando hay un cambio en las necesidades de precisión o en las tasas de producción, en cuyo caso se debe decidir entre el reemplazo integral o parcial y la realización de ajustes. Este caso está relacionado con la obsolescencia debido a los cambios tecnológicos.

A continuación presentaremos tres enfoques aplicados en el análisis económico-financiero; estos son la aplicación del costo anual uniforme equivalente o CAUE, análisis del costo mínimo mediante CAUE y optimización del beneficio.

\subsubsection{Aplicación del CAUE}

Para cada año que transcurre deben observarse las siguientes influencias respecto a un activo o proyecto: el incremento del costo anual de operación o mantenimiento, la disminución del valor de salvamento y la disminución del costo de propiedad debido a la inversión inicial en términos del CAUE. 
Con la finalidad de utilizar los términos aplicados en la Economía, se aplican los conceptos de defensor y retador. Defensor es el activo que la empresa posee actualmente y retador es el activo que lo reemplazará. Para hacer el análisis asumimos el rol del consultor, es decir que no somos propietarios de ninguno de los activos.

Para poder comparar al defensor se necesita pagar el precio actual del mercado de ese activo usado. Para ello se utiliza el valor comercial presente como el costo inicial $\mathrm{C}$ del defensor. Además tendremos un valor de salvamento $\mathrm{S}$, una vida útil $\mathrm{n}$ y un costo anual de operación para el defensor, CAO. Como se utiliza el punto de vista del consultor los datos pueden variar con respecto a los que originalmente tenía la empresa.

El problema queda planteado como la determinación de la decisión más conveniente al final del período, esto es, retener el equipo defensor o reemplazarlo por el retador.

Blank y Tarkin, [2] proponen los ejemplos siguientes:

Supongamos que una empresa tiene un activo comprado hace dos años en $\$ 60000$ y desea retenerlo durante 10 años más. El valor comercial del activo con 2 años de uso es actualmente $\$ 42000$ y el valor comercial de este activo con 12 años de uso es de $\$ 8000$. Los costos anuales de operación, mantenimiento y otros son de $\$ 8000$ por año. El costo de alquiler de un activo similar nuevo es de $\$ 9000$ al año, pagado al finalizar el año, con costos anuales de operación de $\$ 14$ 000. ¿conviene a la empresa el nuevo activo?

\begin{tabular}{|c|c|}
\hline DEFENSOR & RETADOR \\
\hline $\mathrm{C}=42000$ & $\mathrm{C}=42000$ \\
\hline $\mathrm{CAO}=12000$ & $\mathrm{CAO}=14000$ \\
\hline$S=8000$ & 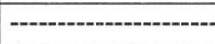 \\
\hline $\bar{N}=10$ Años & $\mathrm{n}=10$ Años \\
\hline
\end{tabular}

$$
\begin{aligned}
\mathrm{CAUE}_{\mathrm{D}} & =\mathrm{C}(\mathrm{A} / \mathrm{C}, \mathrm{i}, \mathrm{n})-\mathrm{S}(\mathrm{A} / \mathrm{M}, \mathrm{i}, \mathrm{n})+\mathrm{CAO} \\
\mathrm{CAUE}_{\mathrm{D}} & =42000(\mathrm{~A} / \mathrm{C}, 0.12,10)-8000(\mathrm{~A} / \mathrm{M}, 0.12,10)+12000 \\
& =18977 \\
\mathrm{CAUE}_{\mathrm{R}} & =9000+14000=23000
\end{aligned}
$$

Por tanto, es más conveniente retener el activo propio.

\subsubsection{Análisis de costo mínimo mediante el CAUE}

El siguiente es un Análisis de Costo Mínimo para determinar mediante este enfoque cuánto tiempo debe permanecer un activo o proyecto en servicio para minimizar su costo total, considerando el valor del dinero en el tiempo y los requerimientos de retorno.

Sea $\mathrm{n}$ el número $\mathrm{p}$ de años que el activo rinde a un mínimo costo anual, es decir, su vida útil económica, que denominaremos "tiempo de permanencia en servicio". 
Trabajando con un procedimiento similar a una simulación determinística para hallar el costo mínimo de vida útil, se va incrementando el valor de la vida útil partiendo de 1 hasta el máximo valor esperado para el activo. Sea k este valor que varía de 1 a $\mathrm{n}$. Para cada valor de $\mathrm{k}$ se determina el CAUE correspondiente.

Además se utilizará:

$S_{\mathrm{k}} \quad$ : Valor de salvamento si el activo tiene $\mathrm{k}$ años

$\mathrm{CAO}_{\mathrm{J}}$ : Costo anual de operación durante el año j; $\mathrm{j}=1,2, \ldots, \mathrm{k}$

Determinación del costo mínimo de un activo:

\begin{tabular}{|c|c|c|c|c|c|}
\hline $\begin{array}{c}\text { VIDA UTIL } \\
\text { K años }\end{array}$ & $\mathbf{S}_{\mathrm{K}}$ & CAO $_{\mathrm{K}}$ & $\begin{array}{c}\text { RECUPERACIÓN } \\
\text { Y RETORNO }\end{array}$ & $\begin{array}{c}\text { COSTOS DE } \\
\text { OPERACIÓN }\end{array}$ & CAUE $_{\mathrm{K}}$ \\
\hline 1 & 9000 & 2500 & 5300 & 2500 & 7800 \\
\hline 2 & 8000 & 2700 & 3681 & 2595 & 6276 \\
\hline 3 & 6000 & 3000 & 3415 & 2717 & 6132 \\
\hline 4 & 2000 & 3500 & 3670 & 2886 & 6556 \\
\hline 5 & 0 & 4500 & 3429 & 3150 & 6579 \\
\hline
\end{tabular}

El menor CAUE corresponde a una vida útil de 3 años, por tanto, de acuerdo con este análisis conviene retener el activo un máximo de 3 años para minimizar el costo total.

\subsubsection{Enfoque de la optimización del beneficio}

Este enfoque plantea el problema de implantar equipos para la producción, evaluar su duración óptima y su substitución, renovación o reemplazo cíclico con la finalidad de extender la actividad productiva más tiempo que el determinado por la duración óptima. Debido a que este proceso se produce en períodos diferentes de tiempo, se toma en consideración el interés compuesto. La consideración del beneficio le da un enfoque real al planteamiento del problema ya que el tomar en cuenta únicamente los costos sólo permite un analisis parcial. El problema se plantea sobre un horizonte anual.

Sean:

CA : Costo de adquisición.

$\mathrm{S}_{\mathrm{n}} \quad$ : Valor de salvamento en el año $n$.

$\mathrm{C}_{\mathrm{n}}$ : Función de costos de operación, mantenimiento y producción.

$\mathrm{I}_{\mathrm{n}} \quad$ : Ingresos obtenidos en el año $\mathrm{n}$.

B : Beneficio acumulado.

El beneficio acumulado actualizado expresado como valor futuro, estará dado por:

$\mathrm{B}=[$ Beneficios Actualizados $]+$ Valor de Salvamento - Cos to de Adquisición .

$B=\sum_{n=1}^{\alpha}\left(1_{n}-C_{n}\right)(1+i)^{-n}+S_{a}(1+i)^{-n}-C A$.

$\alpha$ : Cantidad de años a calcular para cada opción. 
El problema de selección de la mejor alternativa, así como la optimización de la vida de un equipo destinado a la producción y la optimización de su actividad, que pueda significar el realizar renovaciones o reemplazos, tiene como objetivo no sólo determinar la vida útil del equipo sino la optimización de la actividad productiva en el largo plazo.

En tal sentido, se trata de alcanzar un conjunto de objetivos secundarios tales como producir cierto volumen durante un período dado de modo que pueda requerirse uno o más reemplazos del equipo; optimizar el resultado global dado a través del beneficio por la producción total durante un horizonte determinado y evaluar los resultados de la producción en el largo plazo al conocerse el tipo de interés.

El siguiente es un modelo de producción sin plazo de operación, con equipos reemplazados cíclicamente,

$$
\begin{gathered}
\mathrm{BC}_{\alpha}=\mathrm{B}_{\alpha}+\mathrm{B}_{\alpha}(1+\mathrm{i})^{\alpha}+\mathrm{B}_{\alpha}(1+\mathrm{i})^{-2 \alpha}+\ldots .+\mathrm{B}_{\alpha}(1+\mathrm{i})^{-\mathrm{k} \alpha}+\ldots \\
\mathrm{BC}_{\alpha}=\sum_{k=0}^{\infty} \mathrm{B}_{\alpha}(1+\mathrm{i})^{-\mathrm{k} \alpha} \mathrm{B}_{\alpha}\left[1 /\left(1-(1+\mathrm{i})^{-\mathrm{ka}}\right]=\mathrm{B}_{\alpha}\left[\left(1-(1+\mathrm{i})^{-\mathrm{\alpha}}\right]\right.\right.
\end{gathered}
$$

Donde

a = Longitud de cada ciclo o vida del equipo en años.

$\mathrm{BC}_{\alpha}=$ Beneficio final.

$\mathrm{B}_{\alpha} \quad=$ Beneficio acumulado $\mathrm{y}$ actualizado por cada equipo.

i $\quad=$ Tasa de interés.

Se elegirá la alternativa $\alpha$ que maximice el beneficio.

Otro modelo de producción con plazo de operación y equipos que son reemplazados cíclicamente es dado por:

$$
\mathrm{BC}_{\alpha \beta}=\sum_{k=0}^{\beta-1} \mathrm{~B}(1+\mathrm{i})^{-\mathrm{k \alpha}} \mathrm{B}_{\alpha}\left[\left(1-(1+\mathrm{i})^{-\alpha \beta} /\left(1-(1+\mathrm{i})^{-\alpha}\right]\right.\right.
$$

Donde

$\alpha \quad$ : Longitud de cada ciclo o vida del equipo en años

$\mathrm{BCF}_{\alpha \beta}$ : Beneficio final considerando un plazo pre establecido $\mathrm{F}$, es decir que el equipo deja de producir al final del período $\mathrm{F}$

Para un valor $\mathrm{F}=\mathrm{f} \mathrm{y}, \alpha=\mathrm{a}$ es posible renovar hasta $\beta=(\mathrm{f} / \mathrm{a})$ veces el equipo, dentro del horizonte de tiempo considerado para la producción.

Para evaluar si los modelos de Investigación de Operaciones y los modelos económico-financieros arrojan resultados con las mismas tendencias es necesario realizar un análisis comparativo en situaciones equivalentes. Este estudio esta siendo desarrollado por nosotros y será presentado en una publicación posterior. 


\section{CONCLUSIONES}

Las herramientas de Investigación de Operaciones utilizan con mayor precisión el concepto de optimización para la búsqueda del costo total mínimo que permita tomar la decisión de reemplazo. Éstas, generalmente trabajan sólo con los costos en problemas de reemplazo pero es posible plantear una función objetivo para maximizar los beneficios.

- Cuando se utiliza la Investigación de Operaciones es posible definir políticas de reemplazo, es decir secuencias de decisiones que optimizan el costo total.

- Cuando se utiliza el enfoque económico-financiero se aplica con mayor precisión el concepto de activo y su valor en el tiempo.

- En el enfoque económico-financiero también es posible determinar políticas óptimas de reemplazo bajo alternativas de limitación o no del plazo de operación del equipo, con la ventaja de estimar el valor futuro del equipo al conocerse el tipo de interés.

- El análisis aleatorio asociado al riesgo de falla y al impacto que tiene en la decisión se usa con precisión en las herramientas de Investigación de Operaciones, no así en las económicas.

- Es necesario realizar un análisis comparativo de resultados con casos iguales para las técnicas de Investigación de Operaciones para determinar si arrojan resultados que permiten decisiones semejantes.

\section{BIBLIOGRAFIA}

[1] Bang-Jensen, J.; Gutin, G. Digraphs: Theory, Algorithms and Applications. Springer Verlag, 2001, London.

[2] Blank, L.; Tarquin, A. Ingeniería Económica. Mc Graw Hill, 1992, Colombia.

[3] Brealey, R.; Myers, S. Principios de Finanzas Corporativas. Mc Graw Hill, 2001, Madrid.

[4] Cuatrecasas Arbos, L. Gestión Económico-Financiera de la Empresa. Alfaomega, 2000, México.

[5] Duane D. Investigación en Administración para la toma de decisiones. International Thomson Editores, 2001, México.

[6] Rardin, R. Optimization in Operations Research. Prentice Hall, New Jersey, 1998.

[7] Villaplana, J.P. Reemplazamiento de Equipos e Inflación. Investigación Operativa.-ALIO- Volumen 4, Número 2, Agosto 1994. 